\title{
Putting Together Pieces of the Lateral Septum: Multifaceted Functions and Its Neural Pathways
}

\author{
Candace A. Rizzi-Wise and $\mathbb{D}^{D}$ Dong V. Wang
}

https://doi.org/10.1523/ENEURO.0315-21.2021

Department of Neurobiology and Anatomy, Drexel University College of Medicine, Philadelphia, PA 19129

\begin{abstract}
The lateral septum (LS) is implicated as a hub that regulates a variety of affects, such as reward, feeding, anxiety, fear, sociability, and memory. However, it remains unclear how the LS, previously treated as a structure of homogeneity, exhibits such multifaceted functions. Emerging evidence suggests that different functions of the LS are mediated largely by its diverse input and output connections. It has also become clear that the LS is a heterogeneous region, where its dorsal and ventral poles play dissociable and often opposing roles. This functional heterogeneity can often be explained by distinct dorsal and ventral hippocampal inputs along the LS dorsoventral axis, as well as antagonizing connections between LS subregions. Similarly, outputs from LS subregions to respective downstream targets, such as hypothalamic, preoptic, and tegmental areas, also account for this functional heterogeneity. In this review, we provide an updated perspective on LS subregion classification, connectivity, and functions. We also identify key questions that have yet to be addressed in the field.
\end{abstract}

Key words: anxiety; fear; feeding; hippocampus; lateral septum; memory

\section{Significance Statement}

The lateral septum (LS) is a major relay that connects the hippocampus with various subcortical regions; however, how the LS communicates with these regions and processes relevant information has not been well studied. The past several years has brought a number of publications using multidisciplinary approaches, including optogenetics, electrophysiology, and calcium imaging, to elucidate the neural circuitry and functions of the LS. Here, we summarize and integrate current knowledge about the LS circuitry to inspire further research. We propose that the multifaceted functions of the LS are mainly mediated by its diverse input and output connections, and that LS subregions often antagonize each other in competition for controlling behavioral outputs.

\section{Introduction}

The lateral septum (LS) is a major site that connects the hippocampus with multiple subcortical regions, including the lateral hypothalamic area (LHA), lateral preoptic area

\footnotetext{
Received July 24, 2021; accepted October 30, 2021; First published November 11, 2021.

Acknowledgements: We thank Ashley Opalka for the editing of this manuscript.

Correspondence should be addressed to Dong V. Wang at dw657@ drexel.edu.

https://doi.org/10.1523/ENEURO.0315-21.2021

Copyright (C) 2021 Rizzi-Wise and Wang

This is an open-access article distributed under the terms of the Creative Commons Attribution 4.0 International license, which permits unrestricted use, distribution and reproduction in any medium provided that the original work is properly attributed.
}

(LPO), medial hypothalamic area (MHA), medial preoptic area (MPO), ventral tegmental area (VTA), supramammillary area (SUM), medial septum (MS), nucleus accumbens, periaqueductal gray, etc. (Risold and Swanson, 1997b; Sheehan et al., 2004). Expectedly, this central position enables the LS to integrate a variety of emotional, spatial, and cognitive information for regulating behavioral outputs. Pioneer work investigating the LS revealed two of its important roles: reward and "septal rage". The role in reward is readily evident as animals quickly learn to self-stimulate the LS and continue to do so until they are physically exhausted (Olds and Milner, 1954; Prado-Alcala et al., 1984). Septal rage refers to a series of exaggerated emotional and defensive responses to nonthreatening stimuli after damage to the LS (Brady and Nauta, 1953). Since then, 
A
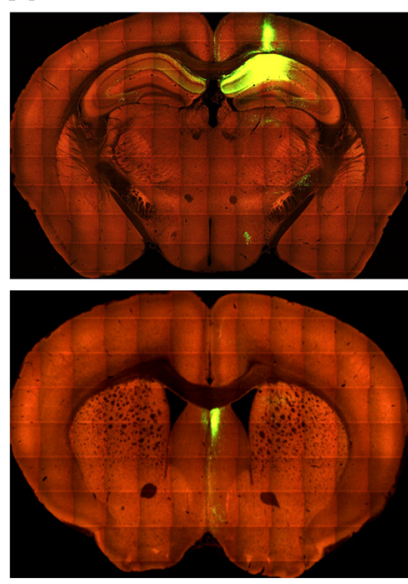

dCA1 --> dm-LS (unilateral)
B
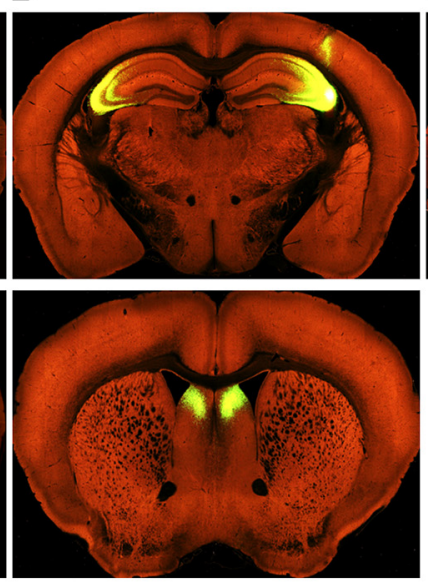

dCA3 --> dl-LS (bilateral)
C
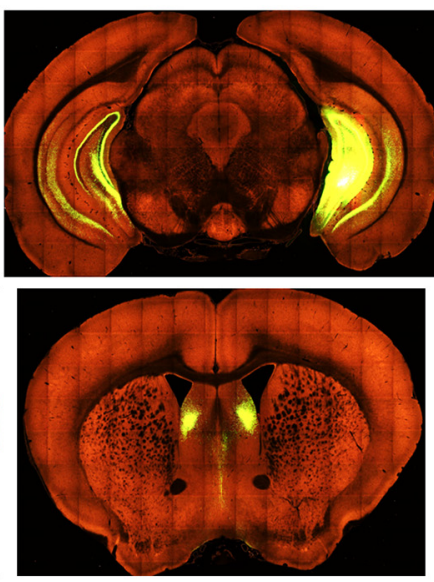

vCA3 --> vl-LS (bilateral)
D
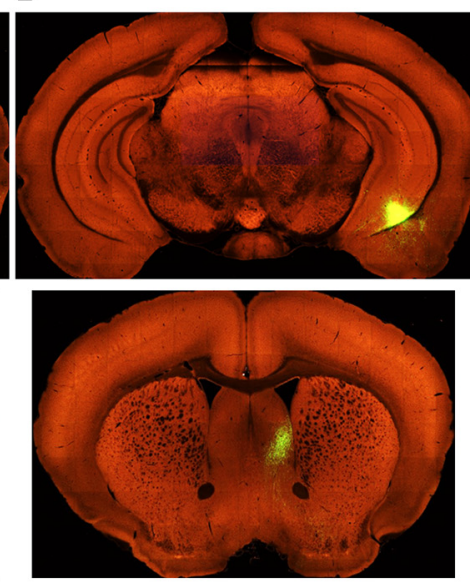

vCA1 --> vm-LS (unilateral)

Figure 1. Subregion specific hippocampus $\rightarrow$ LS projections. $\boldsymbol{A}-\boldsymbol{D}$, top, Coronal brain sections from four individual mice showing unilateral injection (right hemisphere) of AAV-Syn-GFP in the dCA1 $(\boldsymbol{A})$, dCA3 (B), vCA3/DG (C), and vCA1 (D), respectively. Bottom, Coronal brain sections from the same four mice showing the projection in the $\mathrm{dm}$, $\mathrm{dl}$, vl, and vm subregions of the LS, respectively. All brain section photographs are adapted from Allen Brain Atlas - Mouse Connectivity (Oh et al., 2014).

accumulating evidence has evolved to position the LS as a critical structure for many other functions beyond reward and rage.

The LS is composed of predominantly GABAergic neurons, ranging from $85 \%$ to $100 \%$ based on different estimations (Risold and Swanson, 1997a; Zhao et al., 2013; Wong et al., 2016). Correspondingly, most LS neurons express molecular markers for GABAergic neurons: subsets express somatostatin (SST), calbindin or calretinin, but very few express parvalbumin (Zhao et al., 2013; CarusCadavieco et al., 2017; Besnard et al., 2019, 2020). The LS is also enriched with various neuropeptide receptors, including vasopressin, oxytocin, ghrelin, glucagon-like peptide 1 (GLP-1), corticotropin-releasing factor (CRF), neuropeptide $\mathrm{Y}$, and neurotensin receptors (Risold and Swanson, 1997a; Kask et al., 2001; Anthony et al., 2014; Azevedo et al., 2020). Different LS subpopulations appear to express distinct combinations of these neuropeptide markers and, thus, exhibit distinct physiological functions (Risold and Swanson, 1997a; Zhao et al., 2013; Besnard et al., 2019, 2020).

The multifaceted functions of the LS are also a direct result of its diverse input/output connections (Risold and Swanson, 1997b; Oh et al., 2014; Tsanov, 2018). The LS receives the densest input from the hippocampus (Sheehan et al., 2004), and accordingly, we attempted to divide the LS into four subregions solely based on their inputs from different hippocampal subregions. These include the dorsomedial $(\mathrm{dm})$, dorsolateral (dl), ventrolateral (vl), and ventromedial (vm) LS, which preferably receive inputs from hippocampal dCA1/ Sub, dCA3, vCA3, and vCA1/Sub, respectively (Figs. 1, 2). Notably, while the LS receives a unilateral projection from the CA1 (Fig. 1A,D), it receives bilateral projections from the CA3 (Fig. 1B,C). Similarly, outputs from these LS subregions preferentially target distinct subcortical regions (Fig. 2): the dmLS mainly projects to the LHA and LPO; the dl-LS projects to the LHA, LPO, VTA and SUM; and the vI-LS and vm-LS project to the MHA and MPO. On the other hand, there is only sparse projection between the LS and neocortical regions (Risold and Swanson, 1997b; Sheehan et al., 2004). The collection of these works uses a combination of traditional tracing methods and more modern use of viral tracing. While this allows for a solid foundation to build a visualization of comprehensive circuitry, we acknowledge that there are still pathways emerging with the continued study of the LS, particularly using viral tracing techniques, that will need further characterization (Carus-Cadavieco et al., 2017; Jonsson et al., 2017; Chen et al., 2021).

This expansive LS circuitry indicates important subregional differences within the LS, corresponding to diverse or even opposing functions. The opposing function is also supported by findings that LS subregions can directly inhibit each other (Sheehan et al., 2004; Leroy et al., 2018; Oliveira et al., 2021). During our review of the literature, we were able to differentiate studies that specifically target one of the above four LS subregions; however, others likely target two or more of these subregions. To simplify, we refer to dm-LS and dl-LS collectively as dorsal LS (dLS), vl-LS and vm-LS collectively as ventral LS (vLS), and dl-LS and vl-LS collectively as lateral LS. In cases where three or four subregions are targeted, we refer to them collectively as LS. Only recently, modern tools used for circuitry dissections, such as optogenetics and chemogenetics, have been employed to investigate the diverse functions of LS subregions (Yizhar et al., 2011; Roth, 2016). This review will provide a comprehensive overview of the most recent developments in the knowledge of LS circuitry and function, mainly in rodent models, with a specific focus on reward, feeding, anxiety, fear, sociability, and memory.

\section{LS-Associated Circuitry in Reward}

Findings from self-stimulation studies in rodents provided one of the original deductions for the LS having rewarding and reinforcing properties. Only recently has this 


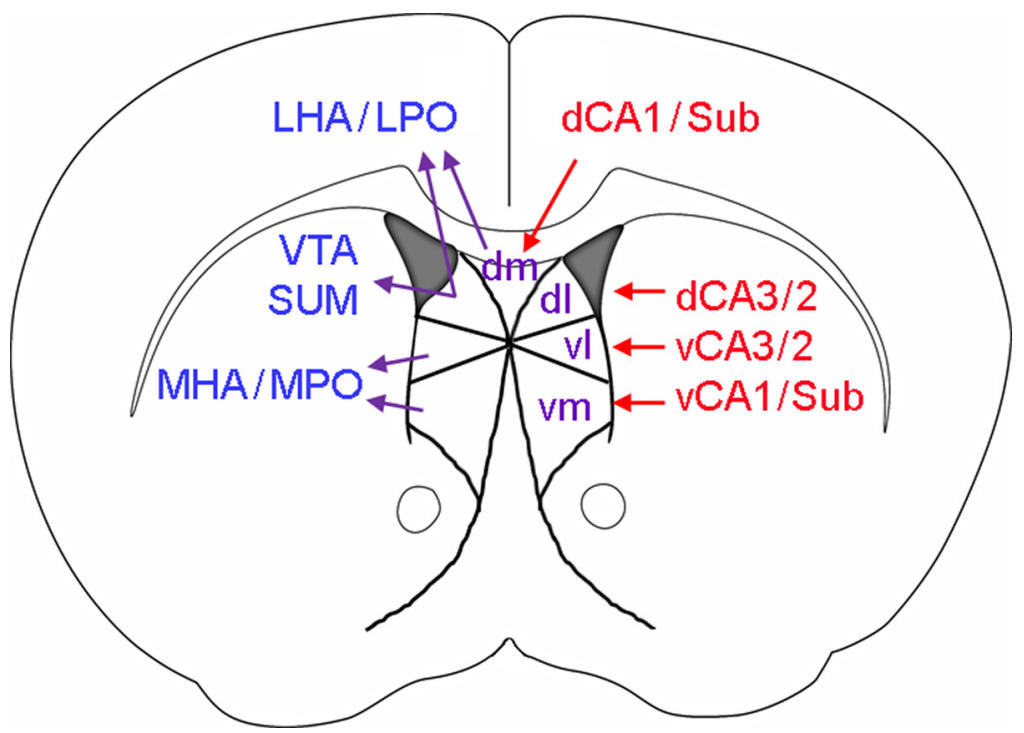

Figure 2. Major LS connections. LS subregions (dm, dl, vl, and vm) and their connections with hippocampal, hypothalamic, and other major areas. CA3/2, CA3 and CA2; d/v, dorsal/ventral; LHA, lateral hypothalamic area; LPO, lateral preoptic area; MHA, medial hypothalamic area; MPO, medial preoptic area; Sub, subiculum; SUM, supramammillary nucleus; VTA, ventral tegmental area.

reward-related function been revisited in the context of LS-associated circuitry involving upstream and downstream connections. Most commonly, experiments studying reward-based behaviors use optogenetic operant conditioning or pharmacological manipulations within cocaine or alcohol use paradigms. These studies indicate that the LS itself can elicit rewarding properties as well as being involved in drug addiction behaviors.

\section{LS and reward}

The pioneering work on the LS demonstrated a repetitive self-stimulation of this region indicative of its role in reward (Olds and Milner, 1954; Prado-Alcala et al., 1984). Building on these early findings, research has hypothesized a connection from the LS to several regions that have been well studied in reward processing, including the VTA, nucleus accumbens, and lateral hypothalamus (Risold and Swanson, 1997b; Sheehan et al., 2004). In support of this, recent studies revealed that pharmacological stimulation of the LS increases VTA dopamine neuronal activity; whereas pharmacological blockade of LS activity decreases dopamine release in the accumbens, indicating a septal control of the VTA in mediating reward (Luo et al., 2011; Jonsson et al., 2017; Vega-Quiroga et al., 2018). Moreover, the lateral LS could directly control the accumbens, a major VTA downstream target, in mediating reward (Zahm et al., 2013; Jonsson et al., 2017). Additionally, a vLS $\rightarrow$ hypothalamic tuberal nucleus $(\mathrm{Tu})$ pathway has been shown to mediate reward as optogenetic activation of this pathway induces real-time place preference (Mu et al., 2020). Together, three parallel LS pathways that engage well-studied rewarding brain areas, namely, dl$\mathrm{LS} \rightarrow \mathrm{VTA}, \mathrm{vLS} \rightarrow \mathrm{Tu}$, and lateral LS $\rightarrow$ accumbens pathways, have been implicated in mediating reward.

\section{LS and drug abuse}

Given that the LS projects directly to the VTA and regulates dopamine activity, it is conceivable that the LS is involved in drug seeking and abuse behaviors. Studies quantifying neuronal activation revealed an increase of immediate early gene expression in the LS after injection or context-induced reinstatement of cocaine (Franklin and Druhan, 2000; Zahm et al., 2010; McGlinchey and AstonJones, 2018). In addition, many LS molecular markers, such as the GLP-1 receptor and cocaine- and amphetamine-regulated transcript peptide, are subject to change after drug intake (Janzsó et al., 2010; Harasta et al., 2015). These changes are indicative that the LS adapts to drug intake and may subsequently affect drug-seeking behaviors. On the other hand, pharmacological inhibition of the LS by baclofen-muscimol injection reduces motivation to indulge in drug-seeking behavior (Pantazis and Aston-Jones, 2020). Additionally, intra-LS injections of opioid receptor antagonists block behavioral sensitization to morphine ( $\mathrm{Li}$ et al., 2021). These findings provide compelling evidence of the LS's role in drug seeking behaviors.

Upstream of the LS, the dorsal hippocampus likely provides contextual information to the dLS for mediating drug seeking behaviors. Corroborating this notion, recent studies revealed a tri-synaptic pathway from the dCA $3 \rightarrow \mathrm{dl}-\mathrm{LS} \rightarrow$ VTA that promotes cocaine seeking (Jiang et al., 2018; Luo et al., 2011). Conversely, chemogenetic inhibition of the dCA $\rightarrow$ dlLS pathway attenuates cocaine seeking (McGlinchey and Aston-Jones, 2018; Werner et al., 2020). Additional evidence suggests that the dLS also recruits downstream LHA to regulate cocaine seeking behaviors (Sartor and AstonJones, 2012). On the other hand, the role of the ventral hippocampus $\rightarrow$ vLS pathway in drug abuse remains unknown. It is possible that this ventral pathway indirectly regulates drug seeking behavior, given that the ventral 
hippocampus plays a critical role in stress and anxiety, and that such negative emotions increase risk of drug seeking and relapse (Sinha, 2001; Fanselow and Dong, 2010). In summary, two dLS-associated pathways, namely, $\mathrm{dCA} 3 \rightarrow \mathrm{dl}-\mathrm{LS} \rightarrow$ VTA and dLS $\rightarrow$ LHA pathways, have been implicated in drug seeking and abuse. Besides processing drug rewards, the LS is also involved in processing natural rewards such as those associated with feeding, which is discussed below.

\section{LS-Associated Circuitry in Feeding}

The LS has long been thought to play a role in feeding given its dense projection to the hypothalamus, a central site that controls appetite and satiety (Risold and Swanson, 1997b). This notion is also supported by the LS's role in reward and emotion, often linking food consumption with eating disorders. Feeding is a complex process that involves psychological, physiological, and behavioral mechanisms. Indeed, available evidence supports the LS's involvement in multiple aspects of feeding behaviors, including food motivation, food intake, and taste preference.

\section{LS and food motivation}

An important aspect of feeding behavior is food motivation, which reflects an effort of food seeking but not necessarily food intake. Food motivation is often studied using operant responding paradigms, in which increased responding (e.g., lever press or nose poke) is interpreted as high motivation for food. Studies that have examined food motivation focus on the function of several receptors in the LS, particularly the GLP-1 and ghrelin receptors, both of which are critical in controlling feeding behavior (Steinert et al., 2017). These receptors appear to exhibit opposite effects: intra-dLS administrations of GLP-1 and its antagonist suppress and increase operant responding for food, respectively (Terrill et al., 2016, 2019), whereas an intra-dLS administration of ghrelin increases operant responding for food (Terrill et al., 2018). Notably, no effect was observed when the same GLP-1 manipulations targeted the intermediate regions of the LS (Terrill et al., 2016). This supports the notion that any role in food motivation is mainly, if not exclusively, confined to the dLS. In addition, the SST-positive neurons in the LS have been shown to drive food motivation (Carus-Cadavieco et al., 2017). Whether these SST-positive and ghrelin (or GLP$1 R$ ) neurons are overlapping or exclusive populations within the LS has yet to be investigated. At the circuit level, optogenetic activation (at $\gamma$ frequency) and inhibition of the infralimbic cortex (IL) $\rightarrow$ LS pathway increases and decreases food motivation, respectively (CarusCadavieco et al., 2017). Overall, the role of the LS in food motivation is in line with the general role of the LS in driving reward and motivation (Olds and Milner, 1954).

\section{LS and food intake}

Separate from the motivation component of feeding, the LS can also directly regulate food intake through pharmacological manipulation of different receptors. Ghrelin, $\mu$-opioid, and GLP-1 receptors all influence food intake: activation of the former two receptors promotes food intake, whereas activation of the latter suppresses it (Terrill et al., 2016, 2018; Calderwood et al., 2020). What remains unclear is how targeting these receptors affects LS activity. At the circuit level, projection-specific manipulations that involve the upstream hippocampus and downstream hypothalamus provide further information about LS connections involved in food intake. First, optogenetic activation and inhibition of the dCA3/hilus $\rightarrow$ dLS/MS pathway reduces and increases food intake, respectively (Azevedo et al., 2019). Second, optogenetic activation of the vCA3 $\rightarrow$ vl-LS pathway reduces food intake (Sweeney and Yang, 2015). Third, optogenetic activation and inhibition of the paraventricular nucleus of the hypothalamus $(P V N) \rightarrow$ VLS pathway reduces and increases food intake, respectively ( $X u$ et al., 2019). These results indicate a suppressive role of both dLS and vLS subregions and their upstream connections in food intake. Downstream of the LS, optogenetic activation of the dLS $\rightarrow$ LHA pathway also reduces food intake (Sweeney and Yang, 2016; Azevedo et al., 2020; Mu et al., 2020). Together, at least four LS-associated pathways, namely, the $\mathrm{dCA} 3 \rightarrow \mathrm{dl}-\mathrm{LS}, \quad \mathrm{vCA} 3 \rightarrow \mathrm{vl}-\mathrm{LS}, \quad \mathrm{PVN} \rightarrow \mathrm{VLS}$, and $\mathrm{dLS} \rightarrow \mathrm{LHA}$ pathways, appear to be exclusively involved in suppression of food intake. Additionally, the regulation of food intake can also be achieved through modification of taste preference, as optogenetic inhibition of the LHA $\rightarrow$ LS pathway enhances sweet taste preference (Fu et al., 2019). One potential concern is that the reduction on food intake could be confounded by an upregulation of negative affects (Xu et al., 2019). However, ample evidence argues against this possibility: pharmacological activation of LS GLP-1 receptors affects food intake but not anxiety (Terrill et al., 2016); similarly, chemogenetic activation of LS vGAT or NTS neurons affects food intake but not anxiety (Sweeney and Yang, 2016; Azevedo et al., 2020). These results suggest that the LS's role in suppressing food intake is largely independent of promoting negative affects. On the other hand, a dissociable role of the LS in regulating negative affects including anxiety and stress has been well studied and is discussed below.

\section{LS-Associated Circuitry in Anxiety}

Mounting evidence also supports a role of the LS in anxiety and stress. To characterize anxiety-like behaviors in rodents, a battery of behavioral paradigms has been developed, including but not limited to open field (OF), light-dark box (LD), elevated plus maze (EPM), novel object, and shock probe tests. In these tests, a decreased time exploring the center area of the OF, the light compartment of the LD, open arms of the EPM, novel objects, or an increased time burying the shock probe, is often considered to be associated with anxiety and stress. Utilization of these paradigms has revealed subregional and molecular profile differences within the LS that allows it to be capable of both promoting and suppressing anxiety-like behaviors.

\section{LS and anxiety promotion}

A variety of stressors can induce LS neuronal activation, evidenced by increased immediate early gene expression 
(Sheehan et al., 2004). Electrophysiology results provide further evidence that most dLS neurons increase activity under anxiogenic conditions on exploration of the open arms of an EPM relative to the enclosed arms (Thomas et al., 2013). Additionally, optogenetic activation and inhibition of a subset of lateral LS neurons (that express CRF2 receptors) promotes and suppresses multiple anxiety-like behaviors, respectively (Anthony et al., 2014). Projectionspecific manipulation indicates that these CRF2 neurons preferentially project to the anterior hypothalamic area (AHA) and promote anxiety via an upregulation of blood corticosterone levels (Anthony et al., 2014). Upstream of the LS, two input regions, namely, the PVN and IL, have been shown to regulate LS activity and promote anxiety-like behaviors as well. Specifically, optogenetic activation of the $\mathrm{PVN} \rightarrow \mathrm{VLS}$ pathway promotes stress-related grooming and escape behaviors (Xu et al., 2019), while optogenetic activation of the $\mathrm{IL} \rightarrow \mathrm{LS}$ pathway decreases exploration of the center area of the OF or open arms of the EPM (Chen et al., 2021). Together, three LS-associated pathways, namely the $\mathrm{PVN} \rightarrow \mathrm{VLS}, \mathrm{IL} \rightarrow \mathrm{LS}$, and lateral LS (CRF2 neurons) $\rightarrow \mathrm{AHA}$ pathways, have been identified to play a key role in promoting anxiety-like behaviors. On the other hand, the LS has also been implicated in suppressing anxiety-like behaviors, which is discussed below.

\section{LS and anxiety suppression}

In contrast to CRF's role in promoting anxiety/stress, intra-LS administration of neuropeptide $Y$ decreases anxiety-like behaviors, evidenced by increased social interaction and other active coping behaviors (Kask et al., 2001; Trent and Menard, 2011). Additionally, activation of the LS serotonin $1 \mathrm{~A}$ receptor reduces stress and promotes active coping behavior during a forced swimming test (Singewald et al., 2011). At the circuit level, available evidence suggests that ventral hippocampal efferents to the LS plays a key role in reducing anxiety-like behaviors. This is evidenced by chemogenetic activation and inhibition of LS-projecting vCA1/vCA3 neurons resulting in a decrease and increase in overall anxiety-like behaviors, respectively (Parfitt et al., 2017). Consistently, the LS-projecting vCA1 neurons are activated when animals battle against anxiogenic conditions, on exploring the open arms of an EPM (Wang et al., 2011; Kosugi et al., 2021). Together, these findings indicate a role of the $\mathrm{vCA} 1 \rightarrow \mathrm{VLS}$ and $\mathrm{vCA} \rightarrow \mathrm{VLS}$ pathways in suppressing anxiety-like behaviors.

These seemingly conflicting roles of the LS in both promoting and suppressing anxiety infer heterogeneity in LS subpopulations. Whether this can be attributed to differences of subregions, neuron types, or pathways remains to be fully elucidated, though available evidence points to the opposite roles of dLS versus VLS in promoting and suppressing anxiety-like behaviors, respectively. A closely related yet distinguishable affect to anxiety is fear, which the LS also has strong ties to. While anxiety is a long-lasting state that persists despite the absence of a specific threat, fear is often elicited by an immediate danger or threat (Davis, 1992; Rosen and Schulkin, 1998).

\section{LS-Associated Circuitry in Fear}

Seminally, damage to the LS resulted in septal rage characterized by increased fear responses to harmless stimuli, which led to the hypothesis that the LS plays an important role in regulating fear (Brady and Nauta, 1953). Follow-up research largely pointed to the LS's role in suppression or relief of fear (Thomas et al., 1991; Sheehan et al., 2004). However, latest research revealed that the LS is capable of both suppressing and promoting fear responses, depending on subregion differences. Most of the research employed a fear conditioning procedure that required animals to learn the association between a cue/ context and a fearful stimulus (e.g., footshock), and freezing response was widely used as an indication of fear.

\section{LS and fear suppression}

While lesion studies often disrupted large portion of the LS, application of more selective techniques, such as optogenetics, have identified a major role of the lateral subregion in suppressing fear. A key finding is that the dl-LS and hippocampal CA3 neurons exhibit highly correlated activation under fearful conditions; in particular, increased dl-LS activity immediately precedes the reduction of freezing responses, indicating a dCA $\rightarrow$ dl-LS pathway in the suppression of fear (Besnard et al., 2019). Similarly, optogenetic activation of VCA3 terminals in the vl-LS also suppresses freezing responses on exposure to a fearful context (Besnard et al., 2020). Further in vivo calcium imaging work identified a subpopulation of LS SST-positive neurons whose activity precedes the reduction of freezing responses (Besnard et al., 2019). Together, these results provide compelling evidence that both the $\mathrm{dCA} 3 \rightarrow \mathrm{dl}-\mathrm{LS}$ and $\mathrm{vCA} 3 \rightarrow \mathrm{vl}$-LS pathways, which likely recruit SST-positive LS neurons, play a key role in the suppression of fear.

\section{LS and fear promotion}

In opposition to the role of the dCA3/vCA3 associated LS pathways in suppressing fear, contrasting studies point to the role of the dCA $1 \rightarrow \mathrm{dm}$-LS pathway in promoting fear. First, transection of the CA1/Sub projection to the LS to anatomically disrupt this pathway abolishes fear responses on exposure to a fearful context or cue (Hunsaker et al., 2009). Second, optogenetic inhibition of the $d C A 1 / S u b \rightarrow d m$-LS pathway decreases freezing responses on exposure to a fearful context (Opalka and Wang, 2020). These results provide initial evidence of the role of the dCA1/Sub $\rightarrow$ dm-LS pathway in promoting fear, though more evidence is needed to confirm this notion. On the other hand, whether the vCA $1 \rightarrow \mathrm{vm}$-LS pathway suppresses fear remains to be validated. Nonetheless, the above findings update the original hypothesis of the LS's role in fear suppression by extending to include fear promotion as well.

To date, there is a lack of understanding of how the LS regulates physiological conditions induced by fear, whereas it has been shown that the LS's involvement in anxiety can be mediated by increased blood corticosterone levels (Anthony et al., 2014). Through its dense 
projections to various hypothalamic nuclei, it is plausible that the LS regulates increases in stress hormones and blood pressure that manifests into fear responses. On the other hand, most studies on LS use similar foot shock paradigms and freezing as a measure of fear. While this is a well-established method, it does limit the interpretations of the results to this specific type of associative fear. In fact, classical LS lesion studies also reported other fear related behavioral changes such as fear aggression (Brady and Nauta, 1953).

\section{LS-Associated Circuitry in Social Behavior}

Indicative of the LS having a role in social behaviors, it is enriched with receptors for two neuropeptides, vasopressin and oxytocin, both of which are important modulators of social behaviors (Donaldson and Young, 2008). Emerging evidence suggests that the LS plays a key role in a variety of social behaviors, including social aggression (e.g., attack, dominance), social fear, mate bonding, family bonding, and social play. The LS circuitry associated with aggression has begun to be revealed; however, the LS-associated circuitry in other social behaviors remains largely unstudied.

\section{LS and social aggression}

Septal rage animals exhibit exaggerated defensive aggression toward conspecifics, indicating a role of the LS in social aggression (Sheehan et al., 2004). Recent studies provide further evidence in dissecting the downstream and upstream connections of the LS in mediating social aggression. Downstream of the LS, the ventromedial hypothalamus $(\mathrm{VMH})$ is established as the center for eliciting social aggression, evidenced by an immediate onset of attack behavior against an intruder in direct response to optogenetic activation of VMH neurons (Lin et al., 2011). Expectedly, optogenetic activation and inhibition of the lateral LS $\rightarrow \mathrm{VMH}$ pathway, which inhibits and activates the $\mathrm{VMH}$, decreases and increases social aggression, respectively (Wong et al., 2016). Upstream of the LS, two hippocampal subregions, namely, the CA2 and vCA1, are known for their social functions (Hitti and Siegelbaum, 2014; Okuyama et al., 2016). Building off this information to include pathway specificity, optogenetic activation of the CA2 $\rightarrow$ lateral LS pathway, which ultimately activates the $\mathrm{VMH}$ via a dLS $\rightarrow$ VLS disinhibition mechanism, promotes social aggression (Leroy et al., 2018). Together, these findings indicate a key role of the $\mathrm{CA} 2 \rightarrow \mathrm{dLS} \rightarrow \mathrm{VLS} \rightarrow \mathrm{VMH}$ pathway in promoting social aggression. It should be noted that this conclusion derives from studies exclusively on male rodents; paradoxically, in female rats, social aggression is associated with activation of the vLS and suppression of the dLS (Oliveira et al., 2021). This opposite role of the dLS versus vLS in male and female social aggression remains to be reconciled and thus needs further investigation.

\section{LS and other social behaviors}

In addition to aggression, another conflictive social behavior is social fear. One characterization of social fear is by pairing an investigation of a conspecific with a footshock. Using such paradigm, it was found that intra-LS administration of oxytocin increased the amount of time spent investigating a social conspecific despite being previously associated with a footshock (Zoicas et al., 2014). This indicates a critical role of LS oxytocin in the suppression of social fear.

Social behaviors can also be cooperative when there is a beneficial interaction between conspecifics. Using such paradigms, lesioning of the LS disrupted kinship behavior and family bonding, indicating a key role of the LS in social bonding (Clemens et al., 2020). Related to neuropeptides, intra-LS administration of vasopressin in male prairie voles facilitates mate bonding, whereas blocking vasopressin receptors eliminates this behavior (Liu et al., 2001). Manipulation of the vasopressin and oxytocin systems in the LS also modulates social play and social approach (Bredewold et al., 2014; Kelly et al., 2020). Together, these findings indicate an important role of the LS in mate bonding, family bonding, social play, and social fear. The neural pathways that support these social behaviors are unclear; however, the LS likely receives oxytocin and vasopressin from the PVN and bed nucleus of the stria terminalis (BNST), respectively, in executing these social functions (De Vries and Buijs, 1983).

\section{LS-Associated Circuitry in Memory}

Given the critical role of the LS in fear and social experiences, it is conceivable that the LS also engages in associated fear and social memory processes. However, despite being a major target of hippocampal efferents, the LS has not been well established for a potential role in memory processes. Until quite recently, evidence that equivalently supports this notion started to emerge. Overall, the LS has been shown to play an important role in each of the memory stages, including memory acquisition (encoding), memory consolidation (transformation of recent memory into long-term memory), and memory retrieval/expression. Most of the relevant studies use a fear conditioning or social investigation paradigm, which will be the focus of the following discussion.

\section{LS and fear memory}

Growing evidence supports the role of the LS in fear memory processes. First, the LS is critical for fear memory acquisition, as disruption of LS activity during fear conditioning training impairs subsequent memory performance, evidenced by reduced freezing responses on re-exposure to footshock chambers (Desmedt et al., 1999; Calandreau et al., 2007, 2010; Hunsaker et al., 2009; Opalka and Wang, 2020). Notably, this role of the LS in acquisition appears to be dependent on its input from the hippocampus (Hunsaker et al., 2009; Opalka and Wang, 2020). Second, the LS is critical for fear memory consolidation, as a posttraining manipulation of the LS (intra-LS administration of CRF) immediately after an inhibitory avoidance task enhances relevant fear memory (Lee, 1995); however, more studies are needed to confirm this notion. Third, the LS is critical for fear memory retrieval/expression, as disruption of LS inputs from the hippocampus during fear memory 
retrieval impairs memory performance (Hunsaker et al., 2009; Besnard et al., 2019, 2020; Opalka and Wang, 2020). One potential concern of this finding is locomotor effects, which confounds interpretation of fear memory that often utilizes freezing behavior as a readout. However, none of these studies found significant changes in locomotion on manipulation of hippocampal efferents to the LS, which is also consistent with the notion of its lack of role in initiating locomotion or running (Bender et al., 2015). Together, these results provide compelling evidence that the LS plays a critical role in fear memory acquisition, consolidation, and likely retrieval/expression.

\section{LS and social memory}

Another line of evidence that supports the role of LS in memory processes is derived from studies on social behavior. A post-training intra-LS administration of either vasopression or oxytocin immediately after a social interaction experience enhances relevant social memory (Dantzer et al., 1988; Popik et al., 1992). Conversely, a post-training intra-LS administration of the vasopression or oxytocin receptor blocker impairs relevant social memory (Dantzer et al., 1988; Everts and Koolhaas, 1997; Lukas et al., 2013). These results provide compelling evidence of the LS's role in social memory consolidation. On the other hand, whether the LS is involved in social memory acquisition remains unclear, as the available, yet limited, evidence is mixed. One study reported that intra-LS administration of a vasopressin or oxytocin receptor blocker immediately before a social interaction impairs the pair bond formation (Liu et al., 2001). In contrast, another study reported that intra-LS administration of an oxytocin receptor blocker does not affect social memory formation (Lukas et al., 2013). Lastly, whether the LS is involved in social memory retrieval/expression has not been investigated.

\section{LS and spatial navigation}

Spatial navigation is dependent on the hippocampus and thus widely used in memory related studies. In particular, hippocampal place cells that encode specific spatial positions are believed to form a cognitive map of the environment (O'Keefe and Nadel, 1978). Like hippocampal place cells, a subset of LS neurons, albeit in a smaller percentage, have been shown to exhibit place coding properties (Zhou et al., 1999; Leutgeb and Mizumori, 2002; Takamura et al., 2006; Wirtshafter and Wilson, 2020; van der Veldt et al., 2021). In general, LS place cells are described as of lesser quality than hippocampal place cells, often with larger or non-focalized place fields. However, not all studies have successfully identified place cells in the LS, which may be because of recording from different LS subregions (Tingley and Buzsáki, 2018). Rather, some LS neurons transform hippocampal spatial information via a phase-coding strategy, that is, these neurons code place fields by firing at specific phases of hippocampal theta oscillation (Tingley and Buzsáki, 2018). In addition, LS neurons also appear to support spatial navigation by incorporating self-motion information such as speed,
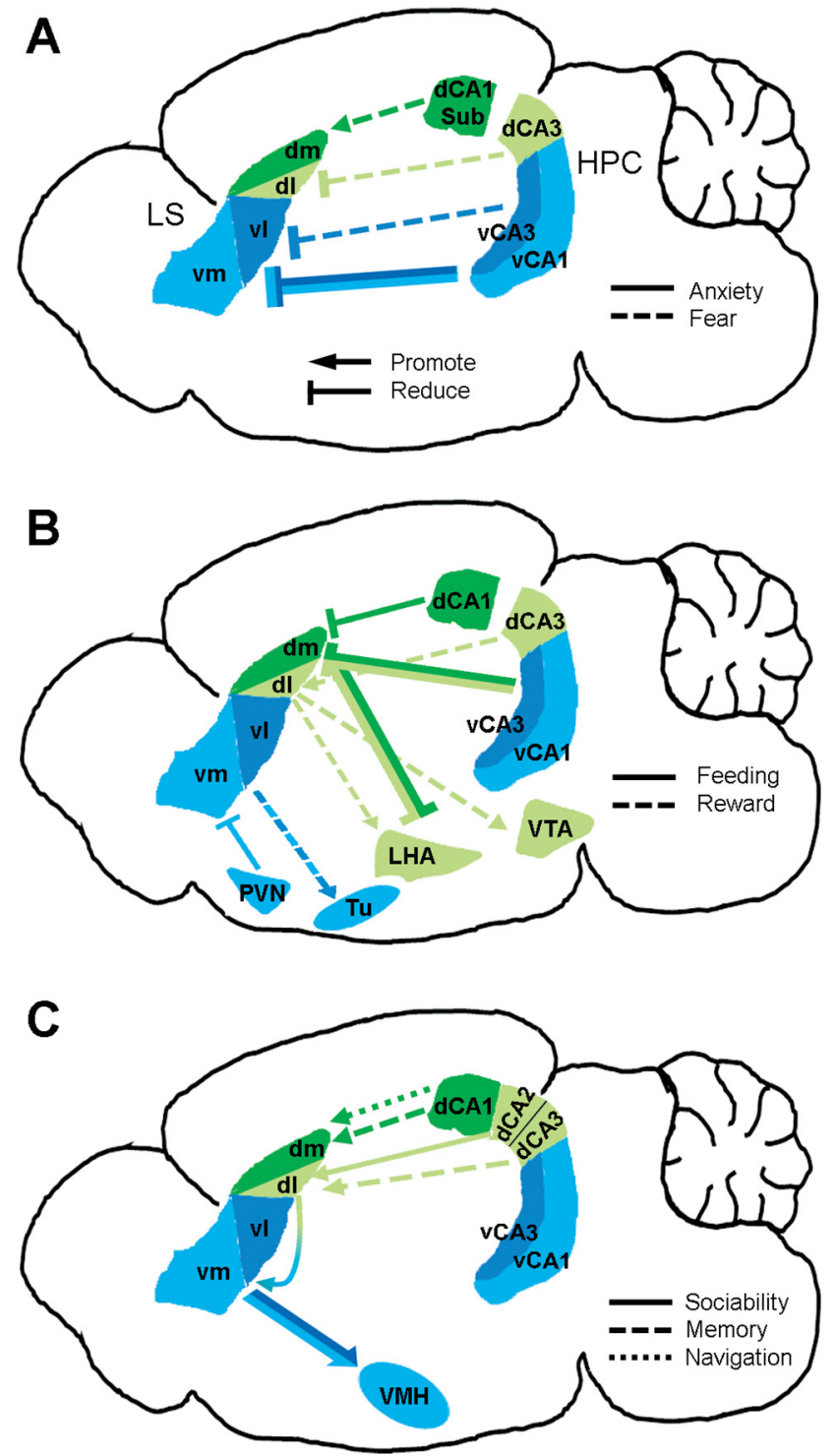

Figure 3. LS subregion associated neural circuits and functions. $\boldsymbol{A}-\boldsymbol{C}$, Major LS subregion connections in fear/anxiety $(\boldsymbol{A})$, reward/feeding $(\boldsymbol{B})$, and other functions $(\boldsymbol{C})$. Note: results from studies that do not differentiate LS subregions are not shown. LS, lateral septum; LHA, lateral hypothalamic area; HPC, hippocampus; PVN, paraventricular nucleus of the hypothalamus; Sub, subiculum; Tu, hypothalamic tuberal nucleus; VMH, ventromedial hypothalamus; VTA, ventral tegmental area.

acceleration, and direction (Wirtshafter and Wilson, 2020; van der Veldt et al., 2021). Together, these studies provide considerable amount of evidence in supporting the LS's role in spatial information coding and likely memoryguided spatial navigation.

\section{LS and sharp-wave ripple}

Latest research revealed that the LS displays a fast oscillation, similar to the $\sim 200-\mathrm{Hz}$ hippocampal ripple oscillation that occurs predominantly during slow-wave sleep and plays a key role in memory consolidation (Buzsáki, 
Table 1: Major LS-associated neural circuitry and functions

\begin{tabular}{|c|c|c|c|c|c|c|}
\hline & Reward & Feeding & Anxiety & Fear & Sociability & Memory \\
\hline $\mathrm{dCA} 1 / \mathrm{Sub} \rightarrow \mathrm{dm}-\mathrm{LS}$ & & & & + & & + \\
\hline $\mathrm{dCA} 3 \rightarrow \mathrm{dl}-\mathrm{LS}$ & + & - & & - & & + \\
\hline $\mathrm{vCA} 3 \rightarrow \mathrm{vl}-\mathrm{LS}$ & & - & & - & & \\
\hline $\mathrm{IL} \rightarrow \mathrm{LS}$ & & + & + & & & \\
\hline dl-LS $\rightarrow$ VTA & + & & & & & \\
\hline $\mathrm{vLS} \rightarrow \mathrm{Tu}$ & + & & & & & \\
\hline $\mathrm{PVN} \rightarrow \mathrm{VLS}$ & & - & + & & & \\
\hline
\end{tabular}

+, promotion effect; -, suppression effect; AHA, anterior hypothalamic area; IL, infralimbic cortex; LHA, lateral hypothalamic area; PVN, paraventricular nucleus of the hypothalamus; Tu, hypothalamic tuberal nucleus; VMH, ventromedial hypothalamus; VTA, ventral tegmental area.

2015). Importantly, this LS fast oscillation is temporally locked to hippocampal ripple, indicating an information flow that conveys ripple-associated memory information from the hippocampus to LS (Tingley and Buzsáki, 2020). Moreover, many LS neurons display a postripple activation that correlates with ripple amplitude (Wirtshafter and Wilson, 2019; Tingley and Buzsáki, 2020), further supporting a hippocampus $\rightarrow$ LS routing of information flow. Interestingly, hippocampal ripple oscillation appears to control the LS for parrel regulation of whole-body glucose homeostasis and memory consolidation (Tingley et al., 2021). This line of evidence provides initial insight into the neural mechanism of hippocampus $\rightarrow$ dLS communication for memory consolidation.

\section{LS-associated memory circuitry}

Few studies have examined the LS-associated circuitry in memory processes; however, inputs from hippocampal CA3, CA2, CA1 and subiculum, and outputs to hypothalamic and mammillary areas are likely the major connections (Risold and Swanson, 1997b). Indeed, the $\mathrm{dCA} 1 \rightarrow \mathrm{dm}$-LS and $\mathrm{dCA} 3 \rightarrow \mathrm{dl}$-LS pathways have been shown to be involved in fear memory acquisition and discrimination processes, respectively (Besnard et al., 2020; Opalka and Wang, 2020). Previous research largely agree on a hippocampal-neocortical communication in supporting cognitive aspects of memory functions (Shohamy and Turk-Browne, 2013). We propose that the hippocampus $\rightarrow$ LS communication accounts for non-cognitive aspects of memory functions that mediate physiological changes, such as stress hormones and blood pressure to prepare the animal for a predicted immediate danger. These non-cognitive responses may be mediated by a few LS downstream regions including the hypothalamus and mammillary areas (Roy et al., 2017).

\section{Conclusions}

In the current review, we consolidated the multifaceted functions of the LS in reward, feeding, anxiety, fear, sociability, spatial navigation, and memory (for other functions such as thermoregulation, cardioregulation, and pain, please see Sheehan et al., 2004). These functions have been mapped in association with distinct LS subregion connections and are summarized below (Fig. 3). This is an exhaustive list of LS functions, but each is not mutually exclusive (Table 1). While each pathway has its own function, multiple pathways could be activated at any given time. These parallel pathways sometimes antagonize each other via the reciprocal GABAergic connections between LS subregions (Sheehan et al., 2004; Leroy et al., 2018; Oliveira et al., 2021). Overall, the multifaceted functions of the LS seem to have a commonality in that they are related to the context or environment of the animal. This often has to do with the salience of a stimulus or other environmental changes, being either positive or negative valence. We propose that the LS as a whole could influence behavior by continuously combining and integrating contextual information from all LS subregions. This communication between LS subregions is likely essential for flexible assigning valence to contextual elements, which need constant updating in an ever-changing environment for animals to make appropriate responses.

\section{LS subregions and major functions}

Previous studies have delineated the LS into subregions mainly based on cytoarchitectural factors and/or molecular markers that do not specifically relate to its connectivity (Risold and Swanson, 1997b; Sheehan et al., 2004). While those previous subregional insights are important for understanding the LS, our proposed classification of LS subregions gives rise to functionally relevant and more clear pathway delineations involving the LS. Specifically, we characterized the LS into four major subregions, namely, dl-LS, dm-LS, vl-LS, and vm-LS, solely based on its major efferent and afferent connections that are also translated to distinct functions.

In the dorsal dimension, the dl-LS has been well studied for its role in reward and drug abuse. Manipulation of this subregion influences VTA dopamine activity, thus regulating various reward-seeking and drug-seeking behaviors (Luo et al., 2011; Jiang et al., 2018; McGlinchey and Aston-Jones, 2018; Werner et al., 2020). Moreover, this promotion of reward is in line with another role of the dlLS in suppressing negative affect such as fear (Besnard et al., 2019). On the other hand, the dm-LS is less well studied. Available evidence suggests that the dm-LS and dl-LS exhibit opposing functions in promoting and 
suppressing learned fear, respectively (Hunsaker et al., 2009; Besnard et al., 2019; Opalka and Wang, 2020). This difference could be explained by the finding that the $\mathrm{dm}$ LS is predominantly involved in memory processes (via its inputs from the dCA1/Sub), whereas the dl-LS is involved in both memory (via its inputs from the dCA3) and affective processes (via its output to VTA), with the affect component often masking the memory component. Additionally, studies that target either the dl-LS or entire dLS exhibit similar effects in suppressing feeding (Sweeney and Yang, 2016; Azevedo et al., 2020; Mu et al., 2020).

In the ventral dimension, the vl-LS and vm-LS exhibit similar functions. First, activation of either or both subregions reduces fear and anxiety (Parfitt et al., 2017; Besnard et al., 2020). Second, activation of either or both subregions reduces social aggression (Leroy et al., 2018). Third, activation of either or both subregions reduces feeding (Sweeney and Yang, 2015; Xu et al., 2019). These findings indicate a general role of the vLS in suppressing negative affect, including fear, anxiety, aggression, and those associated with hunger state. Moreover, this suppression of negative affect is in line with another role of the vLS in promoting reward (Mu et al., 2020).

Collectively, three LS subregions, namely, the dl-LS, vlLS, and vm-LS subregions, exhibit largely similar functions in promoting reward while suppressing negative affect. However, there seem to be differences: while the dl-LS directly, the vl-LS and vm-LS indirectly promotes reward (that is, anti-fear or anti-anxiety). Conversely, while the vl-LS and vm-LS directly, the dl-LS indirectly suppresses negative affect (that is, pro-reward). Additionally, the similar roles of the three subregions in suppressing feeding could be explained by that the vl-LS and vm-LS directly suppress hunger state, whereas the dl-LS promotes reward and thus, may indirectly alleviate hunger. On the other hand, the dm-LS exhibits opposite functions compared with the above three subregions, which could be explained by its major role in supporting memory processing rather than regulating affect. Nonetheless, the functional differentiation of LS subregions has yet to be fully revealed, as the regions needs to be more closely studied.

\section{Outlook}

Recent studies have provided an important division between LS subregions; however, further circuit mapping using targeted techniques are required to fully determine the roles of each subregion in the discussed functions as well as determine whether specific projections involve distinct or overlapping populations of LS neurons. It has also become increasingly clear that the LS is not simply a relay structure; rather, it actively integrates hippocampal and subcortical information, often taking advantage of subregion coordination within the LS, before passing on to downstream regions. Future work that employs neuronal recording techniques such as in vivo electrophysiology and calcium imaging are particularly necessary to shed light on how individual LS neurons and their communications within and outside of the LS contribute to emotion, cognition, behavior, and action selection. Additionally, further work that investigates LS subregional connectivity and interactions should provide new and important insights into the functional heterogeneity as well as integrity of the LS in regulation of effect and behavioral outputs.

\section{References}

Anthony TE, Dee N, Bernard A, Lerchner W, Heintz N, Anderson DJ (2014) Control of stress-induced persistent anxiety by an extraamygdala septohypothalamic circuit. Cell 156:522-536.

Azevedo EP, Pomeranz L, Cheng J, Schneeberger M, Vaughan R, Stern SA, Tan B, Doerig K, Greengard P, Friedman JM (2019) A role of Drd2 hippocampal neurons in context-dependent food intake. Neuron 102:873-886.e5.

Azevedo EP, Tan B, Pomeranz LE, Ivan V, Fetcho R, Schneeberger M, Doerig KR, Liston C, Friedman JM, Stern SA (2020) A limbic circuit selectively links active escape to food suppression. Elife 9: e58894.

Bender F, Gorbati M, Cadavieco MC, Denisova N, Gao X, Holman C, Korotkova T, Ponomarenko A (2015) Theta oscillations regulate the speed of locomotion via a hippocampus to lateral septum pathway. Nat Commun 6:8521.

Besnard A, Gao Y, TaeWoo Kim M, Twarkowski H, Reed AK, Langberg T, Feng W, Xu X, Saur D, Zweifel LS, Davison I, Sahay A (2019) Dorsolateral septum somatostatin interneurons gate mobility to calibrate context-specific behavioral fear responses. Nat Neurosci 22:436-446.

Besnard A, Miller SM, Sahay A (2020) Distinct dorsal and ventral hippocampal CA3 outputs govern contextual fear discrimination. Cell Rep 30:2360-2373.5.

Brady JV, Nauta WJ (1953) Subcortical mechanisms in emotional behavior: affective changes following septal forebrain lesions in the albino rat. J Comp Physiol Psychol 46:339-346.

Bredewold R, Smith CJ, Dumais KM, Veenema AH (2014) Sex-specific modulation of juvenile social play behavior by vasopressin and oxytocin depends on social context. Front Behav Neurosci 8:216.

Buzsáki G (2015) Hippocampal sharp wave-ripple: a cognitive biomarker for episodic memory and planning. Hippocampus 25:1073-1188.

Calandreau L, Jaffard R, Desmedt A (2007) Dissociated roles for the lateral and medial septum in elemental and contextual fear conditioning. Learn Mem 14:422-429.

Calandreau L, Desgranges B, Jaffard R, Desmedt A (2010) Switching from contextual to tone fear conditioning and vice versa: the key role of the glutamatergic hippocampal-lateral septal neurotransmission. Learn Mem 17:440-443.

Calderwood MT, Tseng A, Glenn Stanley B (2020) Lateral septum mu opioid receptors in stimulation of feeding. Brain Res 1734:146648.

Carus-Cadavieco M, Gorbati M, Ye L, Bender F, van der Veldt S, Kosse C, Börgers C, Lee SY, Ramakrishnan C, Hu Y, Denisova N, Ramm F, Volitaki E, Burdakov D, Deisseroth K, Ponomarenko A, Korotkova T (2017) Gamma oscillations organize top-down signalling to hypothalamus and enable food seeking. Nature 542:232236.

Chen YH, Wu JL, Hu NY, Zhuang JP, Li WP, Zhang SR, Li XW, Yang JM, Gao TM (2021) Distinct projections from the infralimbic cortex exert opposing effects in modulating anxiety and fear. J Clin Invest 131:e145692.

Clemens AM, Wang H, Brecht M (2020) The lateral septum mediates kinship behavior in the rat. Nat Commun 11:3161.

Dantzer R, Koob GF, Bluthe RM, Le Moal M (1988) Septal vasopressin modulates social memory in male rats. Brain Res 457:143-147.

Davis M (1992) The role of the amygdala in fear and anxiety. Annu Rev Neurosci 15:353-375.

De Vries GJ, Buijs RM (1983) The origin of the vasopressinergic and oxytocinergic innervation of the rat brain with special reference to the lateral septum. Brain Res 273:307-317. 
Desmedt A, Garcia R, Jaffard R (1999) Vasopressin in the lateral septum promotes elemental conditioning to the detriment of contextual fear conditioning in mice. Eur J Neurosci 11:3913-3921.

Donaldson ZR, Young LJ (2008) Oxytocin, vasopressin, and the neurogenetics of sociality. Science 322:900-904.

Everts HG, Koolhaas JM (1997) Lateral septal vasopressin in rats: role in social and object recognition? Brain Res 760:1-7.

Fanselow MS, Dong HW (2010) Are the dorsal and ventral hippocampus functionally distinct structures? Neuron 65:7-19.

Franklin TR, Druhan JP (2000) Expression of Fos-related antigens in the nucleus accumbens and associated regions following exposure to a cocaine-paired environment. Eur J Neurosci 12:20972106

Fu O, Iwai Y, Narukawa M, Ishikawa AW, Ishii KK, Murata K, Yoshimura Y, Touhara K, Misaka T, Minokoshi Y, Nakajima KI (2019) Hypothalamic neuronal circuits regulating hunger-induced taste modification. Nat Commun 10:4560.

Harasta AE, Power JM, von Jonquieres G, Karl T, Drucker DJ, Housley GD, Schneider M, Klugmann M (2015) Septal glucagonlike peptide 1 receptor expression determines suppression of cocaine-induced behavior. Neuropsychopharmacology 40:19691978.

Hitti FL, Siegelbaum SA (2014) The hippocampal CA2 region is essential for social memory. Nature 508:88-92.

Hunsaker MR, Tran GT, Kesner RP (2009) A behavioral analysis of the role of $\mathrm{CA} 3$ and $\mathrm{CA} 1$ subcortical efferents during classical fear conditioning. Behav Neurosci 123:624-630.

Janzsó G, Valcz G, Thuma A, Szoke B, Lendvai Z, Abrahám H, Kozicz T, Halasy K (2010) Cocaine- and amphetamine-regulated transcript (CART) peptide-immunopositive neuronal elements in the lateral septum: rostrocaudal distribution in the male rat. Brain Res 1362:40-47.

Jiang JX, Liu H, Huang ZZ, Cui Y, Zhang XQ, Zhang XL, Cui Y, Xin WJ (2018) The role of CA3-LS-VTA loop in the formation of conditioned place preference induced by context-associated reward memory for morphine. Addict Biol 23:41-54.

Jonsson S, Morud J, Stomberg R, Ericson M, Söderpalm B (2017) Involvement of lateral septum in alcohol's dopamine-elevating effect in the rat. Addict Biol 22:93-102.

Kask A, Nguyen HP, Pabst R, Von Hörsten S (2001) Neuropeptide $Y$ $Y 1$ receptor-mediated anxiolysis in the dorsocaudal lateral septum: functional antagonism of corticotropin-releasing hormone-induced anxiety. Neuroscience 104:799-806.

Kelly AM, Ong JY, Witmer RA, Ophir AG (2020) Paternal deprivation impairs social behavior putatively via epigenetic modification to lateral septum vasopressin receptor. Sci Adv 6:eabb9116.

Kosugi K, Yoshida K, Suzuki T, Kobayashi K, Yoshida K, Mimura M, Tanaka KF (2021) Activation of ventral CA1 hippocampal neurons projecting to the lateral septum during feeding. Hippocampus 31:294-304.

Lee EH (1995) Corticotropin-releasing factor injected into the lateral septum improves memory function in rats. Chin J Physiol 38:125129.

Leroy F, Park J, Asok A, Brann DH, Meira T, Boyle LM, Buss EW, Kandel ER, Siegelbaum SA (2018) A circuit from hippocampal CA2 to lateral septum disinhibits social aggression. Nature 564:213218.

Leutgeb S, Mizumori SJ (2002) Context-specific spatial representations by lateral septal cells. Neuroscience 112:655-663.

Li YL, Wei S, Liu Q, Gong Q, Zhang QJ, Zheng TG, Yong Z, Chen F, Lawrence AJ, Liang JH (2021) Mu-opioid receptors in septum mediate the development of behavioural sensitization to a single morphine exposure in male rats. Addict Biol e13066.

Lin D, Boyle MP, Dollar P, Lee H, Lein ES, Perona P, Anderson DJ (2011) Functional identification of an aggression locus in the mouse hypothalamus. Nature 470:221-226.

Liu Y, Curtis JT, Wang Z (2001) Vasopressin in the lateral septum regulates pair bond formation in male prairie voles (Microtus ochrogaster). Behav Neurosci 115:910-919.
Lukas M, Toth I, Veenema AH, Neumann ID (2013) Oxytocin mediates rodent social memory within the lateral septum and the medial amygdala depending on the relevance of the social stimulus: male juvenile versus female adult conspecifics. Psychoneuroendocrinology 38:916-926.

Luo AH, Tahsili-Fahadan P, Wise RA, Lupica CR, Aston-Jones G (2011) Linking context with reward: a functional circuit from hippocampal CA3 to ventral tegmental area. Science 333:353-357.

McGlinchey EM, Aston-Jones G (2018) Dorsal hippocampus drives context-induced cocaine seeking via inputs to lateral septum. Neuropsychopharmacology 43:987-1000.

Mu MD, Geng HY, Rong KL, Peng RC, Wang ST, Geng LT, Qian ZM, Yung WH, Ke Y (2020) A limbic circuitry involved in emotional stress-induced grooming. Nat Commun 11:2261.

O'Keefe J, Nadel L (1978) The hippocampus as a cognitive map. Oxford; New York: Clarendon Press; Oxford University Press.

Oh SW, Harris JA, Ng L, Winslow B, Cain N, Mihalas S, Wang Q, Lau C, Kuan L, Henry AM, Mortrud MT, Ouellette B, Nguyen TN, Sorensen SA, Slaughterbeck CR, Wakeman W, Li Y, Feng D, Ho A, Nicholas E, et al. (2014) A mesoscale connectome of the mouse brain. Nature 508:207-214.

Okuyama T, Kitamura T, Roy DS, Itohara S, Tonegawa S (2016) Ventral CA1 neurons store social memory. Science 353:15361541.

Olds J, Milner P (1954) Positive reinforcement produced by electrical stimulation of septal area and other regions of rat brain. J Comp Physiol Psychol 47:419-427.

Oliveira VEM, Lukas M, Wolf HN, Durante E, Lorenz A, Mayer AL, Bludau A, Bosch OJ, Grinevich V, Egger V, de Jong TR, Neumann ID (2021) Oxytocin and vasopressin within the ventral and dorsal lateral septum modulate aggression in female rats. Nat Commun 12:2900.

Opalka AN, Wang DV (2020) Hippocampal efferents to retrosplenial cortex and lateral septum are required for memory acquisition. Learn Mem 27:310-318.

Pantazis CB, Aston-Jones G (2020) Lateral septum inhibition reduces motivation for cocaine: reversal by diazepam. Addict Biol 25:e12742.

Parfitt GM, Nguyen R, Bang JY, Aqrabawi AJ, Tran MM, Seo DK, Richards BA, Kim JC (2017) Bidirectional control of anxiety-related behaviors in mice: role of inputs arising from the ventral hippocampus to the lateral septum and medial prefrontal cortex. Neuropsychopharmacol 42:1715-1728.

Popik P, Vos PE, Van Ree JM (1992) Neurohypophyseal hormone receptors in the septum are implicated in social recognition in the rat. Behav Pharmacol 3:351-358.

Prado-Alcala R, Streather A, Wise RA (1984) Brain stimulation reward and dopamine terminal fields. II. Septal and cortical projections. Brain Res 301:209-219.

Risold PY, Swanson LW (1997a) Chemoarchitecture of the rat lateral septal nucleus. Brain Res Brain Res Rev 24:91-113.

Risold PY, Swanson LW (1997b) Connections of the rat lateral septal complex. Brain Res Brain Res Rev 24:115-195.

Rosen JB, Schulkin J (1998) From normal fear to pathological anxiety. Psychol Rev 105:325-350.

Roth BL (2016) DREADDs for neuroscientists. Neuron 89:683-694.

Roy DS, Kitamura T, Okuyama T, Ogawa SK, Sun C, Obata Y, Yoshiki A, Tonegawa S (2017) Distinct neural circuits for the formation and retrieval of episodic memories. Cell 170:1000-1012.e19.

Sartor GC, Aston-Jones GS (2012) A septal-hypothalamic pathway drives orexin neurons, which is necessary for conditioned cocaine preference. J Neurosci 32:4623-4631.

Sheehan TP, Chambers RA, Russell DS (2004) Regulation of affect by the lateral septum: implications for neuropsychiatry. Brain Res Brain Res Rev 46:71-117.

Shohamy D, Turk-Browne NB (2013) Mechanisms for widespread hippocampal involvement in cognition. J Exp Psychol Gen 142:1159-1170. 
Singewald GM, Rjabokon A, Singewald N, Ebner K (2011) The modulatory role of the lateral septum on neuroendocrine and behavioral stress responses. Neuropsychopharmacology 36:793-804.

Sinha R (2001) How does stress increase risk of drug abuse and relapse? Psychopharmacology (Berl) 158:343-359.

Steinert RE, Feinle-Bisset C, Asarian L, Horowitz M, Beglinger C, Geary N (2017) Ghrelin, CCK, GLP-1, and PYY(3-36): secretory controls and physiological roles in eating and glycemia in health, obesity, and after RYGB. Physiol Rev 97:411-463.

Sweeney P, Yang Y (2015) An excitatory ventral hippocampus to lateral septum circuit that suppresses feeding. Nat Commun 6:10188.

Sweeney P, Yang Y (2016) An inhibitory septum to lateral hypothalamus circuit that suppresses feeding. J Neurosci 36:11185-11195.

Takamura Y, Tamura R, Zhou TL, Kobayashi T, Tran AH, Eifuku S, Ono T (2006) Spatial firing properties of lateral septal neurons. Hippocampus 16:635-644.

Terrill SJ, Jackson CM, Greene HE, Lilly N, Maske CB, Vallejo S, Williams DL (2016) Role of lateral septum glucagon-like peptide 1 receptors in food intake. Am J Physiol Regul Integr Comp Physiol 311:R124-R132.

Terrill SJ, Wall KD, Medina ND, Maske CB, Williams DL (2018) Lateral septum growth hormone secretagogue receptor affects food intake and motivation for sucrose reinforcement. Am J Physiol Regul Integr Comp Physiol 315:R76-R83.

Terrill SJ, Holt MK, Maske CB, Abrams N, Reimann F, Trapp S, Williams DL (2019) Endogenous GLP-1 in lateral septum promotes satiety and suppresses motivation for food in mice. Physiol Behav 206:191-199.

Thomas E, Yadin E, Strickland CE (1991) Septal unit activity during classical conditioning: a regional comparison. Brain Res 547:303308.

Thomas E, Burock D, Knudsen K, Deterding E, Yadin E (2013) Single unit activity in the lateral septum and central nucleus of the amygdala in the elevated plus-maze: a model of exposure therapy? Neurosci Lett 548:269-274.

Tingley D, Buzsáki G (2018) Transformation of a spatial map across the hippocampal-lateral septal circuit. Neuron 98:1229-1242.e5.

Tingley D, Buzsáki G (2020) Routing of hippocampal ripples to subcortical structures via the lateral septum. Neuron 105:138-149.e5.

Tingley D, McClain K, Kaya E, Carpenter J, Buzsáki G (2021) A metabolic function of the hippocampal sharp wave-ripple. Nature 597:82-86.

Trent NL, Menard JL (2011) Infusions of neuropeptide $Y$ into the lateral septum reduce anxiety-related behaviors in the rat. Pharmacol Biochem Behav 99:580-590.

Tsanov M (2018) Differential and complementary roles of medial and lateral septum in the orchestration of limbic oscillations and signal integration. Eur J Neurosci 48:2783-2794. van der Veldt S, Etter G, Mosser CA, Manseau F, Williams S (2021) Conjunctive spatial and self-motion codes are topographically organized in the GABAergic cells of the lateral septum. PLoS Biol 19: e3001383.

Vega-Quiroga I, Yarur HE, Gysling K (2018) Lateral septum stimulation disinhibits dopaminergic neurons in the antero-ventral region of the ventral tegmental area: role of GABA-A alpha 1 receptors. Neuropharmacology 128:76-85.

Wang DV, Wang F, Liu J, Zhang L, Wang Z, Lin L (2011) Neurons in the amygdala with response-selectivity for anxiety in two ethologically based tests. PLoS One 6:e18739.

Werner CT, Mitra S, Auerbach BD, Wang ZJ, Martin JA, Stewart AF, Gobira PH, lida M, An C, Cobb MM (2020) Neuroadaptations in the dorsal hippocampus underlie cocaine seeking during prolonged abstinence. Proc Natl Acad Sci USA 117:26460-26469.

Wirtshafter HS, Wilson MA (2019) Locomotor and hippocampal processing converge in the lateral septum. Curr Biol 29:3177-3192.e3.

Wirtshafter HS, Wilson MA (2020) Differences in reward biased spatial representations in the lateral septum and hippocampus. Elife 9: e55252.

Wong LC, Wang L, D'Amour JA, Yumita T, Chen G, Yamaguchi T, Chang BC, Bernstein H, You X, Feng JE, Froemke RC, Lin D (2016) Effective modulation of male aggression through lateral septum to medial hypothalamus projection. Curr Biol 26:593-604.

Xu Y, Lu Y, Cassidy RM, Mangieri LR, Zhu C, Huang X, Jiang Z, Justice NJ, Xu Y, Arenkiel BR, Tong Q (2019) Identification of a neurocircuit underlying regulation of feeding by stress-related emotional responses. Nat Commun 10:3446.

Yizhar O, Fenno LE, Davidson TJ, Mogri M, Deisseroth K (2011) Optogenetics in neural systems. Neuron 71:9-34.

Zahm DS, Becker ML, Freiman AJ, Strauch S, Degarmo B, Geisler S, Meredith GE, Marinelli M (2010) Fos after single and repeated self-administration of cocaine and saline in the rat: emphasis on the Basal forebrain and recalibration of expression. Neuropsychopharmacology 35:445-463.

Zahm DS, Parsley KP, Schwartz ZM, Cheng AY (2013) On lateral septum-like characteristics of outputs from the accumbal hedonic "hotspot" of Peciña and Berridge with commentary on the transitional nature of basal forebrain "boundaries." J Comp Neurol 521:50-68.

Zhao C, Eisinger B, Gammie SC (2013) Characterization of GABAergic neurons in the mouse lateral septum: a double fluorescence in situ hybridization and immunohistochemical study using tyramide signal amplification. PLoS One 8:e73750.

Zhou TL, Tamura R, Kuriwaki J, Ono T (1999) Comparison of medial and lateral septal neuron activity during performance of spatial tasks in rats. Hippocampus 9:220-234.

Zoicas I, Slattery DA, Neumann ID (2014) Brain oxytocin in social fear conditioning and its extinction: involvement of the lateral septum. Neuropsychopharmacol 39:3027-3035. 